\title{
Incidence and causes of heparin-induced skin lesions
}

\author{
Marc Schindewolf MD, Svantje Schwaner MD, Manfred Wolter MD, Hartmut Kroll MD, \\ Andreas Recke MD, Roland Kaufmann MD, Wolf-Henning Boehncke MD, \\ Edelgard Lindhoff-Last MD, Ralf J. Ludwig MD
}

Previously published at www.cmaj.ca

\section{ABSTRACT}

Background: Little is known about the incidence and causes of heparin-induced skin lesions. The 2 most commonly reported causes of heparin-induced skin lesions are immune-mediated heparin-induced thrombocytopenia and delayed-type hypersensitivity reactions.

Methods: We prospectively examined consecutive patients who received subcutaneous heparin (most often enoxaparin or nadroparin) for the presence of heparin-induced skin lesions. If such lesions were identified, we performed a skin biopsy, platelet count measurements, and antiplatelet-factor 4 antibody and allergy testing.

Results: We enrolled 320 patients. In total, 24 patients (7.5\%, 95\% confidence interval $[\mathrm{Cl}] 4.7 \%-10.6 \%)$ had heparin-induced skin lesions. Delayed-type hypersensitivity reactions were identified as the cause in all 24 patients. One patient with histopathologic evidence of delayedtype hypersensitivity tested positive for antiplatelet-factor 4 antibodies. We identified the following risk factors for heparin-induced skin lesions: a body mass index greater than 25 (odds ratio [OR] 4.6, 95\% Cl 1.7-15.3), duration of heparin therapy longer than 9 days (OR 5.9, 95\% Cl 1.926.3) and female sex (OR 3.0, 95\% Cl 1.1-8.8).

Interpretation: Heparin-induced skin lesions are relatively common, have identifiable risk factors and are commonly caused by a delayed-type hypersensitivity reaction (type IV allergic response). (ClinicalTrials.gov trial register no. NCT00510432.)

Une version française de ce résumé est disponible à l'adresse www.cmaj.ca/cgi/content/full/cmaj.081729/DCI

$\mathrm{H}$ peparin has been used as an anticoagulant for over 60 years. ${ }^{1}$ Well-known adverse effects of heparin therapy are bleeding, osteoporosis, hair loss, and immune and nonimmune heparin-induced thrombocytopenia. The incidence of heparin-induced skin lesions is unknown, despite being increasingly reported. ${ }^{2-4}$ Heparininduced skin lesions may be caused by at least 5 mechanisms: delayed-type (type IV) hypersensitivity responses, ${ }^{2,4-6}$ immune-mediated thrombocytopenia, ${ }^{3}$ type I allergic reactions ${ }^{7.8}$ skin necrosis ${ }^{9}$ and pustulosis. ${ }^{10}$

Heparin-induced skin lesions may indicate the presence of life-threatening heparin-induced thrombocytopenia ${ }^{11}$ - even in the absence of thrombocytopenia. ${ }^{3}$ There are no data available on the incidence of heparin-induced skin lesions or their causes. Given the rising number of reports of heparin-induced skin lesions and the importance of correctly diagnosing this condition, we sought to determine the incidence of heparininduced skin lesions.

\section{Methods}

\section{Study design and patient recruitment}

In this study, "heparin-induced thrombocytopenia" refers to immune heparin-induced thrombocytopenia unless stated otherwise. We enrolled over a 12-month period (April 2007 to February 2008) patients receiving subcutaneous anticoagulant therapy on an in- or out-patient basis at the Hospital of the Johann Wolfgang Goethe University, Frankfurt, Germany. At monthly intervals, we assessed for eligibility all medical inpatients in the Department of Internal Medicine and all outpatients of the Division of Angiology. We included patients aged 18 years or older who had been taking subcutaneous heparin (unfractionated and low-molecular-weight heparins) for a minimum of 7 days. $^{2}$ We obtained informed consent from each patient. We excluded patients with a history of heparin-induced thrombocytopenia or type I or type IV allergic reactions to heparin. We aimed to include a minimum of 300 patients. We planned to end recruitment after at least 20 patients with heparin-induced skin lesions were enrolled or after 500 patients were enrolled.

The investigation and procedures were approved by the ethics committee of the Johann Wolfgang Goethe University and were registered at ClinicalTrials.gov (NCT00510432).

\section{Study procedures}

Eligible patients were seen by 1 or more of the study investigators. The investigator recorded each patient's age, sex, indica-

From the Departments of Internal Medicine, Division of Angiology (Schindewolf, Lindhoff-Last), and Dermatology (Schwaner, Wolter, Kaufmann, Boehncke, Ludwig), Hospital of the Johann Wolfgang Goethe University, Frankfurt am Main, Germany, the DRK-Blooddonorservice (Kroll), Dessau, Germany, and the Department of Dermatology (Recke), University of Lübeck, Lübeck, Germany

Cite as CMAJ 2009. DOI:10.1503/cmaj.081729 
tion for admission and previous exposure to subcutaneous anticoagulants. For female patients, the inspector asked whether they were pregnant or taking hormonal contraceptives. From each patient's clinical records the investigator obtained information about the patient's current anticoagulant therapy (preparation, duration and dose of anticoagulant therapy), height and weight (to calculate body mass index). A clinical examination was performed of the anticoagulant injection sites, as well as a full-body examination of the skin. If a heparin-induced skin lesion was suspected, a second investigator examined the patient. If required, further diagnostic procedures were performed, including a skin biopsy for hematoxylin-eosin staining, platelet count monitoring, heparin-induced platelet activation test and a enzyme-linked immunosorbent assay (ELISA) to detect antiplatelet-factor 4/heparin antibodies. ${ }^{12}$ If heparininduced thrombocytopenia was excluded, patients with skin lesions underwent allergy testing (prick, epicutaneous, intracutaneous and subcutaneous provocation). Any investigations that needed to be repeated to confirm the diagnosis were performed 1-3 weeks later.

\section{Skin biopsies}

Biopsy samples were obtained from suspected heparininduced lesions under local anesthesia. The samples were stained with hematoxylin-eosin and evaluated by 2 experienced dermatopathologists (M.W. and R.K.). We considered the presence of spongiosis and infiltration with leukocytes (eosinophils and lymphocytes) to indicate a delayed-type

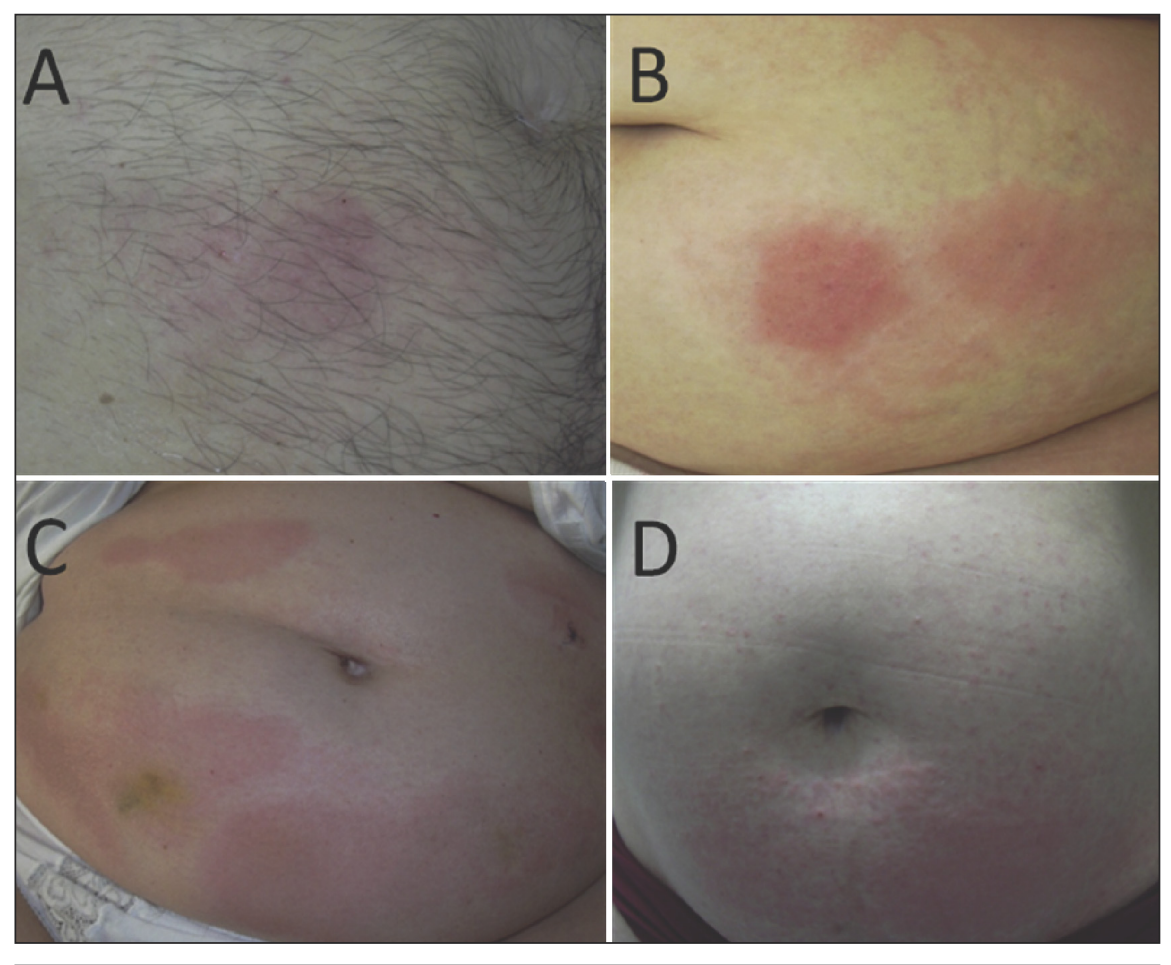

Figure 1: Clinical spectrum of heparin-induced cutaneous delayed-type hypersensitivity responses. A: A red macula with few papules at a heparin-injection site. B: Widespread erythema and red papules originating from heparin-injection sites on the abdomen. C: Generalized red plaques on the entire abdomen. D: Generalized red papules on the entire abdomen originating from heparin injection sites. hypersensitivity reaction. We diagnosed heparin-induced thrombocytopenia if the dermal vessels were occluded.

\section{Allergy testing}

Testing (prick, epicutaneous, intracutaneous and subcutaneous provocation) was initiated no earlier than 6 weeks after resolution of the suspected delayed-type hypersensitivity lesions. We used undiluted original drug formulations as described elsewhere. ${ }^{13}$ The test sites were monitored for development of urticaria 20 minutes after exposure and for eczematous reactions 24, 48 and 96 hours after exposure.

\section{Statistical analysis}

We used $t$ tests or Mann-Whitney rank sum tests to analyze continuous variables and the $\chi^{2}$ test for categorical variables. To adjust for possible correlations between putative risk factors, we performed multiple linear regression analysis.

\section{Results}

\section{Study cohort}

We included 320 out of 325 eligible patients in our analysis. The reasons for the exclusion of 5 patients were history of cutaneous heparin allergy $(n=1)$, aged less than 18 years $(n=1)$, history of heparin-induced thrombocytopenia $(n=1)$ and loss to follow-up $(n=2)$. The mean age of the patients was 60.1 (standard deviation 16.9) years, and equal numbers of men and women were recruited (Table 1). The median duration of heparin therapy was 9 days (range 7-1095 days). Of the included patients, $62.8 \%$ received anticoagulant therapy for prophylaxis and $36.3 \%$ for therapy. The remaining $0.9 \%$ received an anticoagulant initially for prophylaxis and then for therapy. Most patients received either enoxaparin $(59.7 \%)$ or nadroparin $(30.9 \%)$. A few patients received certoparin, tinzaparin, dalteparin or unfractionated heparin for subcutaneous anticoagulant therapy (Appendix 1, available at www.cmaj.ca /cgi/content/full/cmaj.081729/DC2).

\section{Incidence of delayed-type hypersensitivity reactions}

Within the study population, we detected 24 patients with heparin-induced skin lesions, giving an overall incidence of $7.5 \%$ (95\% confidence interval [CI] $4.7 \%-10.6 \%)$. Patients with heparininduced skin lesions mostly had small eczematous plaques at the injection sites. In some cases, widespread lesions were observed, and some patients had generalized, itchy erythematous plaques (Figure 1). Based on the results of a clinical examination, a delayed-type hypersensitivity response was the most likely cause for all the observed lesions. This was 
confirmed for 15 patients by histology and allergy testing, for 5 patients by histology only, and for 3 patients by allergy testing only (Appendix 2, available at www.cmaj.ca/cgi/content /full/cmaj.081729/DC2). One patient did not consent to a punch biopsy or allergy testing. All patients with heparininduced skin lesions were screened for immune-mediated heparin-induced thrombocytopenia by clinical examination and laboratory investigations (platelet count monitoring, heparin-induced platelet activation assay, antiplatelet-factor 4 antibody ELISA). One of the 24 patients who presented with a delayed-type hypersensitivity response to heparin was diagnosed with immune-mediated heparin-induced thrombocytopenia, which had not been considered before study enrollment.

\section{Cross-reactivity among heparin preparations}

In total, 17 patients underwent detailed allergy testing. We observed low cross-reactivity among the tested heparins. Only 2 patients had a positive reaction to a preparation other than the one that had originally triggered the delayed-type hypersensitivity reaction.

\section{Risk factors for delayed-type hypersensitivity reactions}

There were significantly more women in the group with delayed-type hypersensitivity reactions than in the control group (no heparin-induced skin lesions) ( $p=$ 0.028). Patients with heparin-induced delayed-type hypersensitivity were younger than those without delayed-type hypersensitivity responses $(p<0.036)$. Pregnancy $(p<0.001)$, obesity $(p<0.001)$ and a long duration of current heparin therapy $(p<0.001)$, but not previous exposure to heparin $(p=0.08)$, were more common in patients with delayed-type hypersensitivity reactions than patients without these reactions (Table 1).

To adjust for possibly correlated risk factors, we performed multiple linear regression analysis. The presence or absence of heparin-induced skin lesions was predicted from a linear combination of the following independent variables: age greater than 60 years, female, body mass index greater than 25, previous exposure to heparin and duration of current therapy greater than 9 days. Regression analysis confirmed that these were all risk factors, with the exception of pregnancy (Table 2).

\section{Interpretation}

We prospectively evaluated the incidence and causes of heparin-induced skin lesions in a large cohort of patients. The incidence was as high as $7.5 \%$, which is far above the generally expected incidence of $2 \% .{ }^{15}$ During this trial, 9 additional patients with heparin-induced skin lesions were referred to us by colleagues not participating in this investigation. These patients were not included in the cohort of patients presented here. In all study patients, a cutaneous delayed-type hypersensitivity response toward heparin led to the development of skin lesions. Therefore, cutaneous delayed-type hypersensitivity responses should be considered a common adverse reaction to heparin therapy. Delayed-type hypersensitivity reactions appear to be the most common cause of heparin-induced skin lesions, while the other possible pathomechanisms, namely immune-mediated heparin-induced thrombocytopenia, type I allergic reactions, necrosis and pustolosis, are less common.

We had expected to find an incidence of about 1\%-2\% based on the frequency with which we had seen patients with delayed-type hypersensitivity reactions to heparin in the past. During the study we were surprised by the high number of patients with heparin-induced skin lesions. For most of the patients, the diagnosis was made because of our study.
Table 1: Characteristics of the study cohort

\begin{tabular}{|c|c|c|c|c|}
\hline \multirow[b]{2}{*}{$\begin{array}{l}\text { Characteristic; } \\
\text { no. } \% \text { of patients* }\end{array}$} & \multirow[b]{2}{*}{$\begin{array}{l}\text { All patients } \\
n=320\end{array}$} & \multicolumn{2}{|c|}{ Heparin-induced lesions } & \multirow[b]{2}{*}{$\begin{array}{l}\text { Unadjusted } \\
p \text { value }\end{array}$} \\
\hline & & $\begin{array}{c}\text { No } \\
n=296\end{array}$ & $\begin{array}{c}\text { Yes } \\
n=24\end{array}$ & \\
\hline Age, yr, mean (SD) & $60.0(16.9)$ & $60.7(16.4)$ & $53.4(15.4)$ & $0.036 t$ \\
\hline Female & 47.2 & 45.3 & 70.8 & $0.028 \ddagger$ \\
\hline Body mass index (SD) & $26.1(6.4)$ & $25.8(6.4)$ & $29.6(5.9)$ & $<0.001 \dagger$ \\
\hline $\begin{array}{l}\text { Prior exposure } \\
\text { to heparin }\end{array}$ & 69.7 & 68.6 & 83.3 & $0.082 \ddagger$ \\
\hline $\begin{array}{l}\text { Duration of heparin } \\
\text { use, median (range), d }\end{array}$ & $9(7-1095)$ & $9(7-1095)$ & $19(7-336)$ & $<0.001 \dagger$ \\
\hline Pregnant & $9.9 \S$ & $9.0 * *$ & $17.6+t$ & $<0.001 \ddagger$ \\
\hline $\begin{array}{l}\text { Use of oral } \\
\text { contraceptives }\end{array}$ & $6.0 \S$ & $5.2 * *$ & $11.8+\dagger$ & $0.28 \ddagger$ \\
\hline \multicolumn{5}{|c|}{$\begin{array}{l}\text { Note: } S D=\text { standard deviation. } \\
* \text { Unless stated otherwise. } \\
+ \text { Rank sum test. } \\
\neq \chi^{2} \text { test. } \\
\text { §Of the } 151 \text { women in the study population. } \\
* * \text { Of the } 134 \text { women with no heparin-induced skin lesion. } \\
++O f \text { the } 17 \text { women with a heparin-induced skin lesion. }\end{array}$} \\
\hline
\end{tabular}

Table 2: Risk factors for heparin-induced skin lesions*

\begin{tabular}{lccc}
\hline & \multicolumn{2}{c}{$\begin{array}{c}\text { Delayed-type hypersensitivity } \\
\text { reaction; no. of patients }\end{array}$} & \\
\cline { 2 - 3 } Factor & Yes & No & Odds ratio $(95 \% \mathrm{Cl})$ \\
\hline Body mass index $>25$ & 20 & 151 & $4.6(1.7-15.3)$ \\
Female sex & 17 & 134 & $3.0(1.1-8.8)$ \\
Therapy duration $>9$ days & 20 & 136 & $5.9(1.9-26.3)$ \\
\hline
\end{tabular}

Note: $\mathrm{Cl}=$ confidence interval.

*Identified by multiple linear regression analysis that included the following independent variables: age $>60$ years, female sex, body mass index $>25$, prior exposure to heparin, and duration of therapy $>9$ days. 
Therefore, it is tempting to speculate that many cases of heparin-induced skin lesions are undiagnosed. In addition, we identified female sex, obesity and long duration of heparin therapy as risk factors for cutaneous delayed-type hypersensitivity responses to heparin, which is consistent with previous reports. ${ }^{2,4,5,16,17}$ We did not find age or pregnancy to be risk factors, ${ }^{2}$ but few younger or pregnant patients were included in our study, limiting our ability to comment on these factors.

In addition to patient-related risk factors, prospective ${ }^{13}$ and retrospective $^{18}$ data have suggested that the molecular weight of a heparin preparation is a fundamental factor in the determination of its sensitization potential. This might relate to the fact that protein binding of heparins for formation of new epitopes $^{19}$ requires polysaccharide chains of a critical length. ${ }^{20,21}$ The predominant heparins used in our study were enoxaparin and nadroparin, which have almost identical molecular weights. Therefore, we cannot comment on the influence of the molecular weight on the risk of delayed-type hypersensitivity lesions. However, some heparin preparations (e.g., bemiparin) might have a high allergenic potential independent of their molecular weight. ${ }^{18}$ In our study, nadroparin was used less frequently but was associated with more skin reactions than was enoxaparin. It is tempting to speculate that nadroparin possesses an epitope responsible for generation of a delayed-type hypersensitivity response.

We were surprised by the low cross-reactivity of the different anticoagulant preparations in patients with delayedtype hypersensitivity responses toward heparin. This is in contrast to earlier reports from many groups, including ours. ${ }^{2,4,6,7,14,22}$ We now believe that this difference in crossreactivity is because of differences between patients in the earlier studies and in our current study. Previous reports focused on patients who presented to allergy departments for testing of suspected delayed-type hypersensitivity reactions to heparin. Those patients probably had a long course of previous therapy including prolonged exposure to a variety of heparin preparations. ${ }^{23,24}$ In contrast, we actively recruited patients with first-onset delayed-type hypersensitivity reactions to heparin. If a heparin-induced skin lesion was observed, anticoagulant therapy was (most often) changed to fondaparinux. Therefore, among patients recruited for our study, exposure to the anticoagulant inducing a type $1 \mathrm{~V}$ allergic reaction was limited to a much shorter duration, which we believe protected the patients from the development of cross-reactivity.

Our data do not support previously published data that reported an incidence of more than $20 \%$ for heparin-induced thrombocytopenia among 9 patients with heparin-induced skin lesions. ${ }^{11}$ In addition, data from a recently initiated trial investigating the incidence and nature of skin lesions in patients with suspected and confirmed immune heparininduced thrombocytopenia further support our conclusions. We have so far not observed any heparin-associated skin lesions in this cohort of patients (including 11 with immune heparin-induced thrombocytopenia). To thoroughly address the possible association between delayed-type hypersensitivity and immune heparin-induced thrombocytopenia, a study involving patients receiving heparin should assess the presence of delayed-type hypersensitivity and heparininduced thrombocytopenia.

Our findings should lead to an altered view of heparininduced skin lesions. First, physicians should be aware that patients receiving subcutaneous heparin therapy have a high probability of developing a cutaneous delayed-type hypersensitivity response. Therefore, we recommend the routine inspection of heparin injection sites, especially for patients with the identified risk factors. Second, heparin-induced skin lesions should be viewed as a symptom; thus, the underlying cause should be identified. If a heparin-induced skin lesion is observed, we recommend obtaining a punch biopsy, comparing platelet counts before, during and after therapy, and performing appropriate laboratory investigations to exclude heparininduced thrombocytopenia. If heparin-induced thrombocytopenia is excluded, either unfractionated heparin administered intravenously ${ }^{6}$ or fondaparinux administered subcutaneously ${ }^{20,23,24}$ can be used for further anticoagulation. If heparininduced thrombocytopenia is clinically suspected and alternative anticoagulation is required, argatroban, danaparoid or lepirudin can be used until heparin-induced thrombocytopenia is excluded.

\section{Limitations}

A limitation of our study is that we only tested for heparininduced thrombocytopenia in patients with heparin-induced skin lesions. Nevertheless, in our cohort of patients, we observed only 1 patient with delayed-type hypersensitivity and immune heparin-induced thrombocytopenia. However, we do not believe that this points to an association between the diseases, but rather indicates that delayed-type hypersensitivity and heparin-induced thrombocytopenia should be viewed as independent conditions.

\section{Conclusion}

Heparin-induced skin lesions occur frequently in patients receiving subcutaneous heparin therapy. In most cases, heparin-induced skin lesions are caused by a delayed-type hypersensitivity reaction. We identified obesity, female sex and long duration of heparin therapy as patient-related risk factors for development of cutaneous delayed-type hypersensitivity responses toward heparin.

This paper has been peer reviewed.

Competing interests: None declared.

Contributors: Marc Schindewolf and Ralf Ludwig designed the study. Marc Schindewolf, Svantje Schwaner and Ralf Ludwig recruited patients and collected the data. Allergy testing was performed by Wolf-Henning Boehncke. Histology was performed and analyzed by Manfred Wolter and Roland Kaufmann. Hartmut Kroll performed the heparin-induced platelet activation test. Andreas Recke carried out the statistical analysis. Edelgard Lindhoff-Last was responsible for the detection of antiplatelet-factor 4 antibodies. All of the authors were involved in the analyses and interpretation of the data, the writing and revision of the manuscript and gave approval of the final version for publication.

Funding: The study was funded by the Departments of Angiology and Dermatology, Johann Wolfgang Goethe University, Frankfurt am Main, Germany. 


\section{REFERENCES}

1. Alban S. From heparins to factor Xa inhibitors and beyond. Eur J Clin Invest 2005;35:12-20.

2. Ludwig RJ, Schindewolf M, Utikal J, et al. Management of cutaneous type IV hypersensitivity reactions induced by heparin. Thromb Haemost 2006;96:611-7.

3. Warkentin TE, Roberts RS, Hirsh J, et al. Heparin-induced skin lesions and other unusual sequelae of the heparin-induced thrombocytopenia syndrome: a nested cohort study. Chest 2005;127:1857-61.

4. Bircher AJ, Harr T, Hohenstein L, et al. Hypersensitivity reactions to anticoagulant drugs: diagnosis and management options. Allergy 2006;61:1432-40

5. Jappe U. Allergy to heparins and anticoagulants with a similar pharmacological profile: an update. Blood Coagul Fibrinolysis 2006;17:605-13.

6. Gaigl Z, Pfeuffer P, Raith P, et al. Tolerance to intravenous heparin in patients with delayed-type hypersensitivity to heparins: a prospective study. $\mathrm{Br}$ J Haematol 2005; 128:389-92.

7. Utikal J, Peitsch WK, Booken D, et al. Hypersensitivity to the pentasaccharide fondaparinux in patients with delayed-type heparin allergy. Thromb Haemost 2005; 94:895-6.

8. Berkun Y, Haviv YS, Schwartz LB, et al. Heparin-induced recurrent anaphylaxis. Clin Exp Allergy 2004:34:1916-8.

9. Singh S, Verma M, Bahekar A, et al. Enoxaparin-induced skin necrosis: a fatal outcome. Am J Ther 2007;14:408-10.

10. Komericki P, Grims RH, Kränke B, et al. Acute generalized exanthematous pustolosis from dalteparin. J Am Acad Dermatol 2007;57:718-21.

11. Harenberg J, Huhle G, Wang L, et al. Association of heparin-induced skin lesions, intracutaneous tests, and heparin-induced IgG. Allergy 1999;54:473-7.

12. Eichler P, Budde U, Haas S, et al. First workshop for detection of heparin-induced antibodies: validation of the heparin-induced platelet-activation test (HIPA) in comparison with a PF4/heparin ELISA. Thromb Haemost 1999;81:625-9.

13. Ludwig RJ, Schindewolf M, Alban S, et al. Molecular weight determines the frequency of delayed type hypersensitivity reactions to heparin and synthetic oligosaccharides. Thromb Haemost 2005;94:1265-9.

14. Grims RH, Weger W, Reiter H, et al. Delayed-type hypersensitivity to low molec- ular weight heparins and heparinoids: cross-reactivity does not depend on molecuar weight. Br J Dermatol 2007;157:514-7.

15. Bircher AJ, Fluckiger R, Buchner SA. Eczematous infiltrated plaques to subcutaneous heparin: a type IV allergic reaction. Br J Dermatol 1990;123:507-14

16. Ludwig RJ, Beier C, Lindhoff-Last E, et al. Tolerance of fondaparinux in a patien allergic to heparins and other glycosaminoglycans. Contact Dermatitis 2003;49:158-9.

17. Pichler WJ. Delayed drug hypersensitivity reactions. Ann Intern Med 2003; 139:683-93.

18. Bank I, Libourel EJ, Middeldorp S, et al. High rate of skin complications due to low-molecular-weight heparins in pregnant women. J Thromb Haemost 2003; 1:859-61.

19. Mazzolai L, Hohlfeld P, Spertini F, et al. Fondaparinux is a safe alternative in case of heparin intolerance during pregnancy. Blood 2006;108:1569-70.

20. Ludwig RJ, Schindewolf M, Lindhoff-Last E, et al. The influence of heparin's molecular weight and the incidence of delayed type hypersensitivity reactions revisited; in response to Grims et al. Br J Dermatol 2008;158:849-51.

21. Alban S, Greinacher A. Role of sulfated polysaccharides in the pathogenesis of heparin-induced thrombocytopenia. In: Warkentin TE, Greinacher A, editors. Heparin-induced thrombocytopenia. 3rd ed. New York (NY): Marcel Dekker; 2004. p. 197-221

22. Lane DA. Heparin binding and neutralizing proteins. In: Lane DA, Lindahl U, editors. Heparin. 1st ed. London (UK): Edward Arnold; 1989. p. 363-91.

23. Jappe U, Juschka U, Kuner N, et al. Fondaparinux: a suitable alternative in cases of delayed-type allergy to heparins and semisynthetic heparinoids? A study of 7 cases. Contact Dermatitis 2004;51:67-72.

24. Schindewolf M, Ludwig RJ, Wolter M, et al. Tolerance of fondaparinux in patients with generalized contact dermatitis to heparin. J Eur Acad Dermatol Venereo 2008;22:378-80.

Correspondence to: Prof. Ralf J. Ludwig, Department

of Dermatology; University of Lübeck, Ratzeburger Allee 160,

D-23538 Lübeck Germany; fax 49451 500-2981;

ralf.ludwig@uk-sh.de

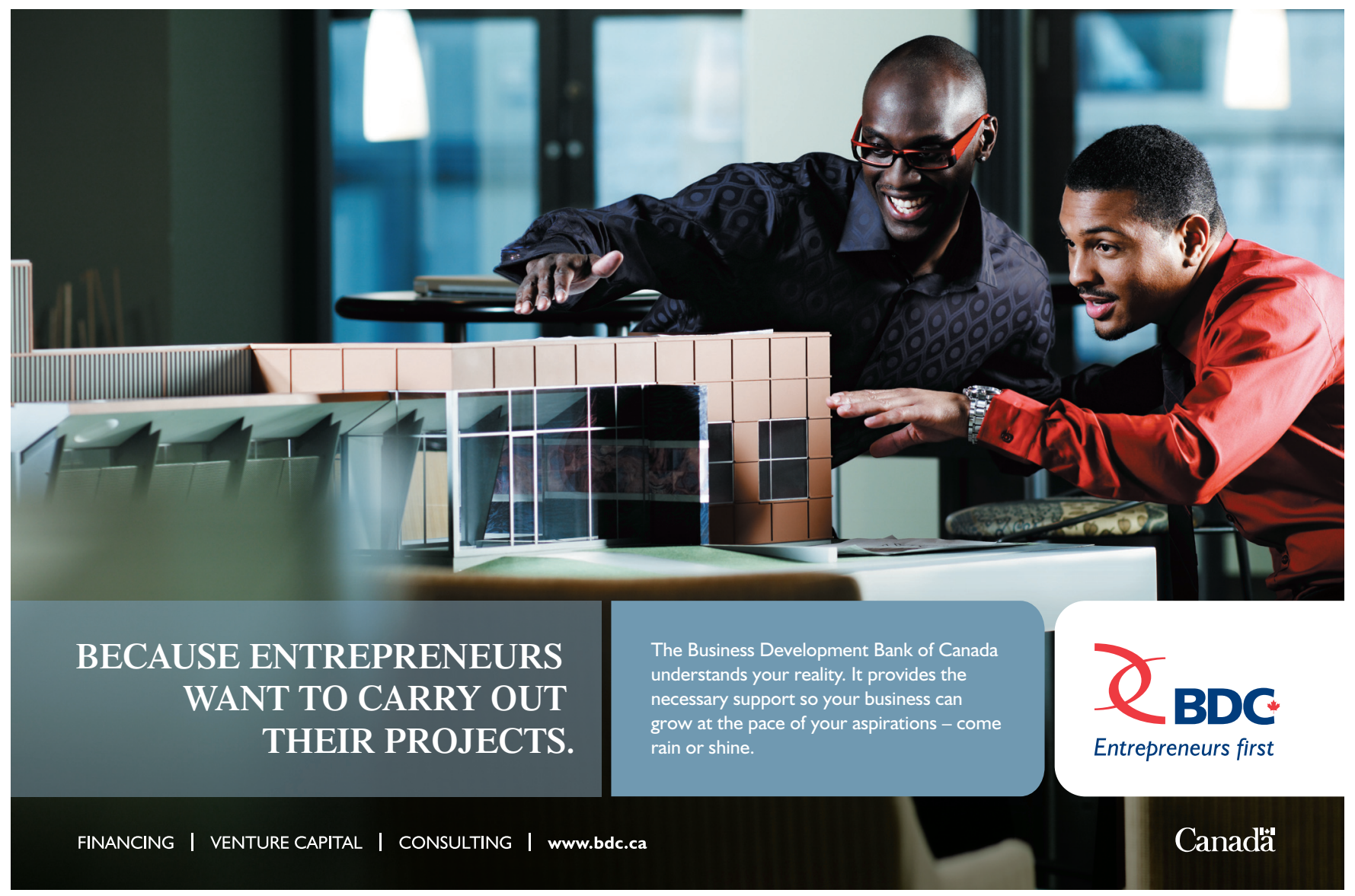

